VOL. $70(2004)$ [397-400]

\title{
A REMARK ON PREINVEX FUNCTIONS
}

\author{
Jianwen Peng and Xianjun Long
}

\begin{abstract}
In this paper, we show that the ratio of preinvex functions is invex. Hence, we give a positive answer to the open question which was proposed in a paper of Yang, Yang and Teo in (2003).
\end{abstract}

\section{INTRODUCTION}

Let $R^{n}$ denotes $n$-dimension Euclidean space. In [2], Hanson considered the real differentiable function $f(x)$ on $R^{n}$ whose gradient $\nabla f(x)$ satisfies the condition: for any $x, y \in R^{n}$, there exists a vector $\eta(x, y) \in R^{n}$ such that

$$
f(x) \geqslant f(y)+\nabla f(y) \eta(x, y) .
$$

Craven [3] called this an invex function. Later, Weir and Mond [4] and Weir and Jeyakumar [5] introduced preinvex functions defined as follows.

Let $K \subset R^{n}$ and $f: K \rightarrow R$. Then $f$ is preinvex if for any $x, y \in K$, there exists a vector $\eta(x, y) \in R^{n}$, for all $\alpha \in[0,1], y+\alpha \eta(x, y) \in K$

$$
f(y+\alpha \eta(x, y)) \leqslant \alpha f(x)+(1-\alpha) f(y) .
$$

It is easy to show that preinvexity is a generalisation of invexity for nondifferentiable function.

In [6], Yang and Chen presented a wider class of generalised convex functions, called semipreinvex functions as follows.

A set $K$ in $R^{n}$ is said to satisfy the "semi-connected" property, if for any $x, y \in K$ and $\alpha \in[0,1]$, there exists a vector $\eta(x, y, \alpha) \in R^{n}$, such that $y+\alpha \eta(x, y, \alpha) \in K$. Let $K$ be a set in $R^{n}$ having the "semi-connected" property with $\eta(x, y, \alpha): K \times K \times[0,1] \longrightarrow R^{n}$ and $f(x)$ be a real function on $K$. Then $f$ is called semi-preinvex with respect to $\eta(x, y, \alpha)$ if for $x, y \in K$ and $\alpha \in[0,1]$,

$$
f(y+\alpha \eta(x, y, \alpha)) \leqslant \alpha f(x)+(1-\alpha) f(y)
$$

Received 13th May, 2004

This research was supported by the National Natural Science Foundation of China (Grant No. 10171118) and Education Committee Item Research Foundation of Chongqing (Grant No. 030801).

Copyright Clearance Centre, Inc. Serial-fee code: 0004-9727/04 \$A2.00+0.00. 
holds and $\lim _{\alpha \downarrow 0} \alpha \eta(x, y, a)=0$.

The following result is due to Khan and Hanson [7] and Craven and Mond [8].

THEOREM 1.1. Let $X_{0} \subset R^{n}$ and let $f$ and $g$ be real-valued functions defined on $X_{0}$. If $f(x) \geqslant 0, g(x)>0, f(x)$ and $-g(x)$ are invex with respect to a same $\eta(x, y)$ on $X_{0}$, then $f(x) / g(x)$ is an invex function with respect to $\bar{\eta}(x, y)=(g(y) / g(x)) \eta(x, y)$.

Yang, Yang and Teo [1] generalise Theorem 1.1 as follows.

TheOREM 1.2. (See [1, Theorem 2.9].) Let $X_{0} \subset R^{n}$ and let $f$ and $g$ be realvalued differential functions defined on $X_{0}$. If $f(x) \geqslant 0, g(x)>0, f(x)$ and $-g(x)$ are semipreinvex with respect to a same $\eta(x, y, \alpha)$ on $X_{0}$, and $\lim _{\alpha \rightarrow 0} \eta(x, y, \alpha)=\eta(x, y)$, then $f(x) / g(x)$ is an invex function with respect to $\bar{\eta}(x, y)=(g(y) / g(x)) \eta(x, y)$.

Then, Yang, Yang and Teo [1] proposed an open question as follows:

Is there a similar result as that of Theorem 1.2 for preinvex functions?

In this paper, we show that the ratio of preinvex functions is invex. Hence, we give a positive answer to the open question in [1].

\section{Main Results}

First of all, we prove the following result which is a generalisation of Theorem 1.1 and a similar result with $[1$, Theorem 2.8$]$.

THEOREM 2.1. Let $X_{0} \subset R^{n}$ and let $f$ and $g$ be real-valued functions defined on $X_{0}$. If $f(x) \geqslant 0, g(x)>0, f(x)$ and $-g(x)$ are preinvex with respect to a same $\eta(x, y)$ on $X_{0}$, then $f(x) / g(x)$ is a semipreinvex function with respect to $\eta^{*}(x, y, \alpha)$ $=[(g(y)) /(\alpha g(y))+(1-\alpha) g(x)] \eta(x, y)$.

Proof: Since $f(x)$ and $-g(x)$ are preinvex with respect to a same $\eta(x, y)$ and $f(x) \geqslant 0, g(x)>0$, we have, for all $x, y \in X_{0}$ and $\alpha \in[0,1], y+\alpha \eta^{*}(x, y, \alpha) \in X_{0}$, and

$$
\begin{aligned}
\left(\frac{f}{g}\right)(y & \left.+\alpha \eta^{*}(x, y, \alpha)\right) \\
& =\frac{f\left(y+\alpha \eta^{*}(x, y, \alpha)\right)}{g\left(y+\alpha \eta^{*}(x, y, \alpha)\right)} \\
& =\frac{f(y+[(\alpha g(y)) /(\alpha g(y)+(1-\alpha) g(x))] \eta(x, y))}{g(y+[(\alpha g(y)) /(\alpha g(y)+(1-\alpha) g(x))] \eta(x, y))} \\
& \leqslant \frac{(\alpha g(y)) /(\alpha g(y)+(1-\alpha) g(x)) f(x)+((1-\alpha) g(x)) /(\alpha g(y)+(1-\alpha) g(x)) f(y)}{(\alpha g(y)) /(\alpha g(y)+(1-\alpha) g(x)) g(x)+((1-\alpha) g(x)) /(\alpha g(y)+(1-\alpha) g(x)) g(y)} \\
& =\frac{\alpha g(y) f(x)+(1-\alpha) g(x) f(y)}{\alpha g(y) g(x)+(1-\alpha) g(x) g(y)} \\
& =\frac{\alpha g(y) f(x)+(1-\alpha) g(x) f(y)}{g(x) g(y)} \\
& =\alpha \frac{f(x)}{g(x)}+(1-\alpha) \frac{f(y)}{g(y)}
\end{aligned}
$$




$$
=\alpha\left(\frac{f}{g}\right)(x)+(1-\alpha)\left(\frac{f}{g}\right)(y)
$$

That is, $f(x) / g(x)$ is a semipreinvex function with respect to $\eta^{*}(x, y, \alpha)$.

The following result gives a positive answer to the open question in [1].

THEOREM 2.2. Let $X_{0} \subset R^{n}$ and let $f$ and $g$ be real-valued differential functions defined on $X_{0}$. If $f(x) \geqslant 0, g(x)>0, f(x)$ and $-g(x)$ are preinvex with respect to a same $\eta(x, y)$ on $X_{0}$, then $f(x) / g(x)$ is an invex function with respect to $\bar{\eta}(x, y)$ $=[g(y) / g(x)] \eta(x, y)$.

Proof: By Theorem 2.1, we know that $f(x) / g(x)$ is a semipreinvex function with respect to $\eta^{*}(x, y, \alpha)=[(g(y)) /(\alpha g(y)+(1-\alpha) g(x))] \eta(x, y)$. That is, for all $x, y \in X_{0}$ and $\alpha \in[0,1]$,

$$
\left(\frac{f}{g}\right)\left(y+\alpha \eta^{*}(x, y, \alpha)\right) \leqslant \alpha\left(\frac{f}{g}\right)(x)+(1-\alpha)\left(\frac{f}{g}\right)(y) .
$$

Then,

$$
\frac{(f / g)\left(y+\alpha \eta^{*}(x, y, \alpha)\right)-(f / g)(y)}{\alpha} \leqslant\left(\frac{f}{g}\right)(x)-\left(\frac{f}{g}\right)(y) .
$$

Let $\alpha \rightarrow 0$, and note that $\lim _{\alpha \rightarrow 0} \eta^{*}(x, y, \alpha)=\bar{\eta}(x, y)$, we have

$$
\nabla\left(\frac{f}{g}\right)(y) \bar{\eta}(x, y) \leqslant\left(\frac{f}{g}\right)(x)-\left(\frac{f}{g}\right)(y) .
$$

Hence, $f(x) / g(x)$ is an invex function with respect to $\bar{\eta}(x, y)$.

\section{REFERENCES}

[1] X.M. Yang, X.Q. Yang and K.L. Teo, 'On properties of semipreinvex function', Bull. Austral. Math. Soc. 68 (2003), 449-459.

[2] M.A. Hanson, 'On sufficiency of the Kuhn-Tuker conditions', Math. Anal. Appl. 80 (1981), 544-550.

[3] B.D. Craven, 'Invex functions and constrained local minima', Bull. Austral. Math. Soc. 24 (1981), 357-366.

[4] T. Weir and B. Mond, 'Pre-invex functions in multiple objective optimizaton', J. Math. Anal. Appl. 136 (1988), 29-38.

[5] T. Weir and V. Jeyakumar, 'A class of nonconvex functions and mathematical programming', Bull. Austral. Math. Soc. 38 (1988), 177-189.

[6] X.Q. Yang and G.Y. Chen, 'A class of nonconvex functions and pre-variational inequalities', J. Math. Anal. Appl. 169 (1992), 359-373.

[7] Z.A. Khan and M.A. Hanson, 'On retio of invexity in mathematical programming', $J$. Math. Anal. Appl. 205 (1997), 330-336.

[8] B.D. Craven and B. Mond, 'Fractional programming with invexity', in Progress in optimization, Appl. Optim. 30 (Kluwer Acad. Publ., Dordrecht, 1999), pp. 79-89. 
College of Mathematics and Computer Science

Chongqing Normal University

Chongqing $\mathbf{4 0 0 0 4 7}$

Peoples Republic of China

e-mail: pjw7503_cn@sina.com

and

Department of Mathematics

Inner Mongolia University

Hohhot 010021

Inner Mongolia

Peoples Republic of China
College of Mathematics and Computer Science

Chongqing Normal University

Chongqing 400047

Peoples Republic of China 